\title{
Anisotropic X-Ray Scattering of Transiently Oriented Water
}

\author{
Kyung Hwan Kim, ${ }^{1,2, *}$ Alexander Späh, ${ }^{1}$ Harshad Pathak, ${ }^{1}$ Cheolhee Yang $\odot,{ }^{2}$ Stefano Bonetti® ${ }^{1,3}$ Katrin Amann-Winkel, ${ }^{1}$ \\ Daniel Mariedahl, ${ }^{1}$ Daniel Schlesinger $\odot,{ }^{1,4}$ Jonas A. Sellberg, ${ }^{5}$ Derek Mendez, ${ }^{6}$ Gijs van der Schot $\odot,{ }^{7}$ Harold Y. Hwang, ${ }^{8}$ \\ Jesse Clark, ${ }^{9}$ Owada Shigeki, ${ }^{10}$ Togashi Tadashi, ${ }^{10}$ Yoshihisa Harada $\odot,{ }^{11}$ Hirohito Ogasawara, ${ }^{9}$ Tetsuo Katayama, ${ }^{10}$ \\ Anders Nilsson $\odot,{ }^{1}$ and Fivos Perakis $\oplus^{1, \dagger}$ \\ ${ }^{1}$ Department of Physics, AlbaNova University Center, Stockholm University, SE-10691 Stockholm, Sweden \\ ${ }^{2}$ Department of Chemistry, POSTECH, Pohang 37673, Republic of Korea \\ ${ }^{3}$ Department of Molecular Sciences and Nanosystems, Ca' Foscari University of Venice, 30172 Venice-Mestre, Italy \\ ${ }^{4}$ Department of Environmental Science and Bolin Centre for Climate Research, Stockholm University, 11418 Stockholm, Sweden \\ ${ }^{5}$ Biomedical and X-Ray Physics, Department of Applied Physics, AlbaNova University Center, \\ KTH Royal Institute of Technology, SE-10691 Stockholm, Sweden \\ ${ }^{6}$ Molecular Biophysics and Integrated Bioimaging Division, Lawrence Berkeley National Laboratory, Berkeley, CA 94720, USA \\ ${ }^{7}$ Department of Cell and Molecular Biology, Laboratory of Molecular Biophysics, Uppsala University, SE-75124 Uppsala, Sweden \\ ${ }^{8}$ Department of Chemistry, Massachusetts Institute of Technology, Cambridge, Massachusetts 02139, USA \\ ${ }^{9}$ SLAC National Accelerator Laboratory, Menlo Park, California 94025, USA \\ ${ }^{10}$ Japan Synchrotron Radiation Research Institute, Kouto 1-1-1, Sayo, Hyogo 679-5198, Japan \\ ${ }^{11}$ Institute for Solid State Physics, The University of Tokyo, Kouto 1-1-1, Sayo, Hyogo 679-5148, Japan
}

(Received 28 April 2020; accepted 8 July 2020; published 11 August 2020)

\begin{abstract}
We study the structural dynamics of liquid water by time-resolved anisotropic x-ray scattering under the optical Kerr effect condition. In this way, we can separate the anisotropic scattering decay of $160 \mathrm{fs}$ from the delayed temperature increase of $\sim 0.1 \mathrm{~K}$ occurring at $1 \mathrm{ps}$ and quantify transient changes in the O-O pair distribution function. Polarizable molecular dynamics simulations reproduce well the experiment, indicating transient alignment of molecules along the electric field, which shortens the nearest-neighbor distances. In addition, analysis of the simulated water local structure provides evidence that two hypothesized fluctuating water configurations exhibit different polarizability.
\end{abstract}

DOI: 10.1103/PhysRevLett.125.076002

Understanding the molecular-level dynamics of liquid water is of fundamental importance for a broad range of fields, ranging from biochemical applications to climate research. One of the challenges, however, is that various techniques capture different aspects of the underlying dynamics related to the corresponding experimental observables. In the case of 2D-IR spectroscopy, the observed dynamics arise from changes in the $\mathrm{OH}$ stretch frequency and contain contributions from librations and intermolecular low-frequency modes [1-3]. Complementary information can be obtained by optical Kerr effect (OKE) spectroscopy, which exploits the change of refractive index induced by an electric field in an isotropic medium [4-6]. On the other hand, scattering techniques, such as inelastic ultraviolet scattering [7], quasielastic neutron scattering [8], and inelastic x-ray scattering [9] allow us to capture dynamics in the frequency,

Published by the American Physical Society under the terms of the Creative Commons Attribution 4.0 International license. Further distribution of this work must maintain attribution to the author(s) and the published article's title, journal citation, and DOI. Funded by Bibsam. as well as structural information encoded by the momentum transfer $q$ dependence.

Traditional OKE spectroscopy utilizes a pump-probe scheme, where the electric field of an optical pump induces transient birefringence on a material, and a time-delayed optical probe monitors the dynamics. In the case of liquid water, the reorientation dynamics has been the subject of previous theoretical [10-13] and experimental investigations [14-18]. Upon supercooling, the appearance of two components becomes evident in the low-frequency range $\sim 200 \mathrm{~cm}^{-1}$, which are attributed to two transient local configurations, namely, that arising from high- and lowdensity liquid (HDL and LDL) forms [18]. However, due to the mostly isotropic molecular polarizability of water and the significant instrumental function dependence on the analysis, a complex fitting procedure is required for the physical interpretation of the observed signals. Anisotropic scattering under the OKE condition has not been possible with synchrotron or laboratory hard $\mathrm{x}$-ray sources because capturing relaxation dynamics of water requires femtosecond time resolution and, in addition, resolving the anisotropic component in the diffuse scattering arising from a liquid requires extremely high flux. With the advent ofx-ray free electron laser (XFEL) sources, which provide 


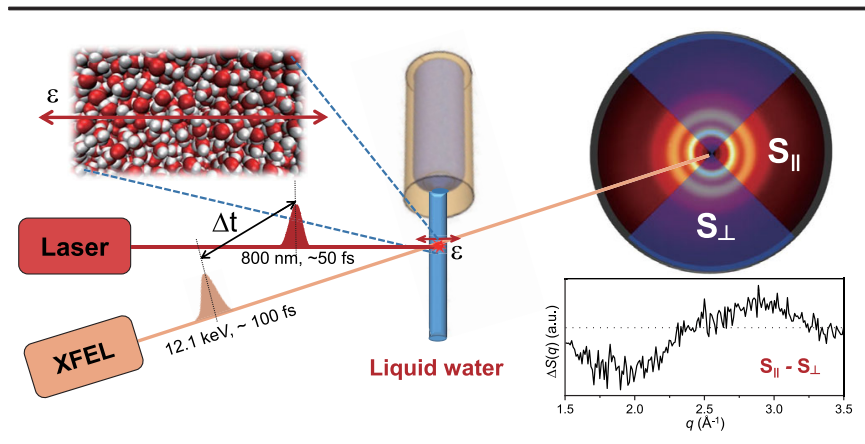

FIG. 1. The experimental setup using anisotropic x-ray scattering under OKE condition. The alignment of liquid water is triggered by a linearly polarized optical laser pulse. Subsequently, a time-delayed $\mathrm{x}$-ray pulse probes the anisotropy decay, obtained by subtracting the perpendicular $\left(S_{\perp}\right)$ from the parallel $\left(S_{\|}\right)$ scattering pattern.

$\sim 10^{12}$ photons per pulse with a sub-100 fs time resolution, anisotropic scattering of liquid water under OKE condition has become possible. Previous investigations using anisotropic scattering measured an aqueous solution of $\left[\mathrm{Co}(\text { terpy })_{2}\right]^{2+}$ and used the result to accurately determine the time zero of their measurement [19].

Here, we demonstrate the implementation of timedependent anisotropic x-ray scattering of liquid water under OKE condition, by replacing the optical probe pulse with an intense $\mathrm{X}$-ray pulse from an XFEL [20,21]. This approach allows us to separate the anisotropic dynamics from the isotropic heating signal arising from a temperature increase of $\sim 0.1 \mathrm{~K}$. Anisotropy develops within our time resolution and decays within $\sim 160 \mathrm{fs,}$, while the heating response increases within $\sim 1$ ps. The observed signal is related to structural changes, as the probed difference in the structure factor reflects changes in the radial distribution function. In other words, the structural dynamics in real space are projected in reciprocal space by the scattering geometry and the ultrafast pulses allow us to resolve any changes on atomic length scales, comparable to the x-ray wavelengths.

The experimental setup is shown in Fig. 1 and the detailed procedures for the data collection are given in the Supplemental Material [22]. A continuous water jet $(T=298 \mathrm{~K})$ of $80 \mu \mathrm{m}$ diameter was generated by an injector in a sample chamber filled with helium. An $800 \mathrm{~nm}$ pump laser pulse (duration $50 \mathrm{fs}$, pulse energy $450 \mu \mathrm{J}$, focus size $200 \mu \mathrm{m}$ ) was utilized to trigger the alignment of the water molecules in the sample along the horizontal electric field of the laser pulse. We have investigated nonlinear absorption effects [23] by varying the fluence that are shown to be negligible (see Supplemental Material [22]). A femtosecond XFEL probe pulse was used to capture the alignment and relaxation dynamics of the sample. The time-dependent twodimensional anisotropic scattering patterns of transiently oriented liquid water were measured at various time delays
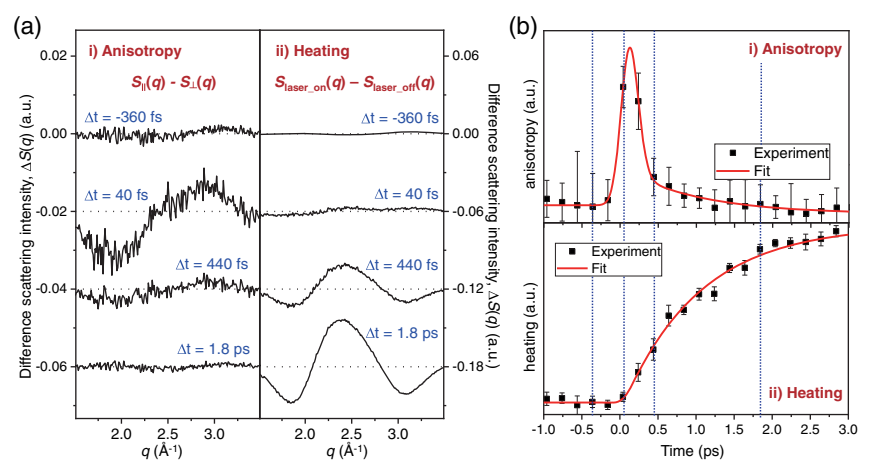

FIG. 2. (a) Experimental difference scattering curves for anisotropy (left, $S_{\|}-S_{\perp}$ ) and heating (right, $S_{\text {laser_on }}-S_{\text {laser_off }}$ ) measured at four different time delays at $298 \mathrm{~K}$. (b) Timedependent changes of anisotropy (top) and heating (bottom) and the corresponding fits. The anisotropy decays biexponentially with the relaxation time of $\sim 160 \mathrm{fs}$ and 1 ps and the heating signal increases with the timescale of $\sim 1$ ps. The four delay points shown in (a) are indicated with blue dashed lines.

ranging from -1 to 100 ps. Because of this analysis scheme, we could separate the anisotropic scattering decay from the delayed isotropic $\mathrm{x}$-ray scattering signal, reflecting the temperature increase [19] (see Supplemental Material [22]). The time-dependent change of anisotropy was examined by comparing the 1D curves of the perpendicular $\left(S_{\perp}\right)$ and parallel $\left(S_{\|}\right)$cuts of the pattern, where the angle of the cuts is defined with respect to the polarization of the pump laser pulse.

The experimental difference scattering curves for anisotropy (left panel, $S_{\|}-S_{\perp}$ ) and heating (right panel, $\left.S_{\text {laser_on }}-S_{\text {laser_off }}\right)$ measured at various time delays and their time-dependent changes are shown in Fig. 2. An offset has been introduced on the $y$ axis to facilitate the comparison between the corresponding signal size of the isotropic and anisotropic components. We note that the shape of the difference scattering curves for anisotropy and heating are very distinct from each other and are also consistent with the previous pump-probe measurements on aqueous solutions $[19,24]$. At negative time delays, the signals from both anisotropy and heating are zero within the noise level, indicating that there are no serious artifacts obstructing our measurements. Immediately after the interaction with the pump laser pulse, the signal from anisotropy is almost fully developed, while that from heating is negligible. At 1 ps time delay, the anisotropic signal is mostly diminished, while the heating signal is steadily increasing and persists up to $100 \mathrm{ps}$. In order to follow the time-dependent changes of the signal amplitude, the modulus of the area under the difference scattering curves is integrated and plotted in Fig. 2(b). The time traces are then fitted with exponential functions convoluted with the instrument response function (IRF). The fitting functions and parameters used in both the experiment and simulation results are presented in detail in the Supplemental Material [22]. As shown in Fig. 2(b), 
anisotropy exhibits a biexponential decay with relaxation times of $160 \mathrm{fs}$ and $1 \mathrm{ps}$, whereas the heating signal increases with a timescale of 1 ps.

We interpret the observed time-resolved signal in the following way: The electric field of the incident pump $800 \mathrm{~nm}$ pulse induces a transient alignment of water molecules, imposed by the polarization of the electric field. The subsequent decay occurs due to the gradual randomization of the induced alignment due to equilibrium dynamics of the water molecules. We note here that the observed signal originates mainly from $\mathrm{O}-\mathrm{O}$ contribution, due to the higher $\mathrm{x}$-ray scattering cross section of oxygen than that of hydrogen, reflected by the different number of electrons. As such, the observed fast dynamics are attributed directly to O-O intermolecular motion, which can be related to the collective low-frequency modes manifesting in the terahertz regime with subpicosecond lifetimes [25]. A slower second anisotropic component arises from structural relaxation and diffusion of water molecules, which results in complete loss of the initial alignment. The energy deposited by the pump pulse is converted to heat by gradual energy relaxation through vibrational modes that can exhibit very strong intra- and intermolecular coupling [26]. The observed molecular alignment is phenomenologically similar with the alignment of water molecules imposed by a constant electric field [27], which also result in an anisotropic $\mathrm{x}$-ray scattering signal or by terahertz laser pulses [28]. However, the difference is that, while water molecules are aligned due to the permanent dipole moment under the constant or terahertz electric field, the induced dipole plays an important role under the OKE condition since the alignment of the permanent dipole cannot follow the fast oscillating field of the pump pulses.

Although our x-ray scattering data contain direct timeresolved structural information on the water molecule dynamics induced by OKE, a direct Fourier transform into real space and subsequent analysis on the $\mathrm{O}-\mathrm{O}$ pair distribution function is not possible due to the limited $q$ range of our experiment. Instead, here the experiment is complemented by Modify to molecuar dyancmis (MD) simulation. So far, there have been several MD simulation studies modeling the OKE signal and the corresponding decay times and temperature dependence [10-13]. However, the simulation of the time-dependent decay of structural correlation has in most cases been attempted without the use of an external electric field. This approach is needed in order to explain our anisotropic scattering data on the laser-driven alignment and reorientation dynamics of water molecules. We have performed MD simulations with the polarizable water model SWM4NDP [29] and the explicit electric field [30] implemented in the GROMACS molecular dynamics package [31]. The computational details are described in the Supplemental Material [22]. We note that the degree of anisotropy was smaller in MD than in the experiment and higher pulse
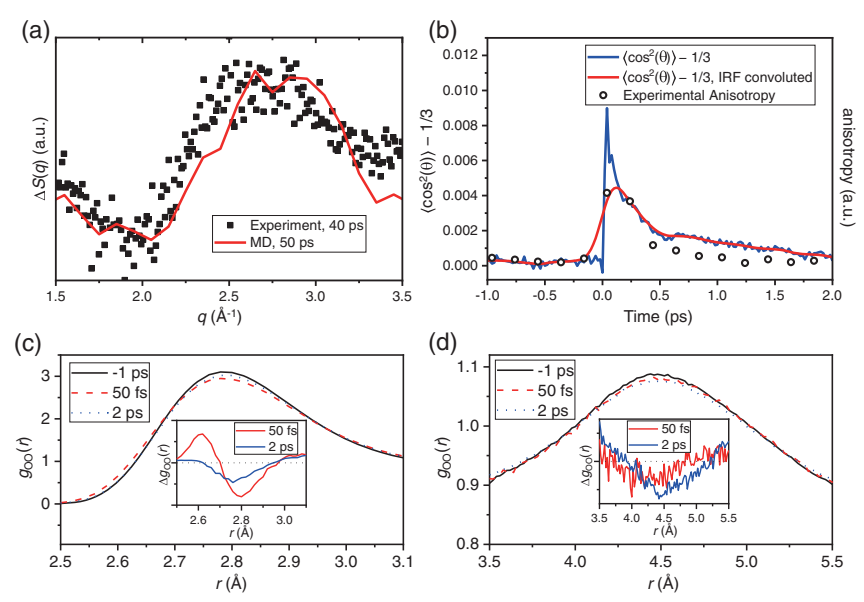

FIG. 3. MD simulation of the transiently oriented water and the comparison with the experimental results. (a) Difference scattering curve for anisotropy calculated from MD simulations at $50 \mathrm{fs}$ after the external electric field (red line) is compared with the experiment (black square). (b) Time-dependent $\left\langle\cos ^{2}(\theta)\right\rangle-1 / 3$ of water molecules in the simulation (blue solid line) is compared with the experimental time-dependent anisotropy (open circle). The angle $\theta$ is the angle between the dipole of the water molecule and the polarization of the external electric field. When $\left\langle\cos ^{2}(\theta)\right\rangle-1 / 3$ is convoluted with the instrument response function ( $\sim 210 \mathrm{fs}$ ), it exhibits qualitatively good agreement with the experiment. (c),(d) The $\mathrm{O}-\mathrm{O}$ radial distribution functions calculated from the MD at -1 ps (solid black line), $50 \mathrm{fs}$ (dashed red line), and 2 ps (dotted blue line) are compared at the first peak (c) and the second peak (d). (Insets) The difference signals.

energy was necessary to get a comparable effect. This observation presumably indicates that the SWM4-NDP water model underestimates the induced dipole under the fast oscillating field. The use of $a b$ initio MD simulation with the external electric field would potentially be able to model the effect more accurately, but such an approach on a system size with hundreds of molecules is challenging and beyond the scope of this Letter. Despite the limitations of the MD simulations, we observe qualitative agreement with the experimental data, and the model assists in the interpretation of the observed signal.

The simulation results and a direct comparison with the experimental data are shown in Fig. 3. The difference scattering curve for anisotropy at $50 \mathrm{fs}$ after the external electric field is calculated from MD simulations and compared with the experimental data in Fig. 3(a). The simulation results exhibit good agreement with the experimental data at $40 \mathrm{fs}$, without using any further adjustment or fitting. This level of agreement provides confidence that detailed structural information on the alignment and reorientation dynamics can be deduced by analyzing the simulation results.

By defining $\theta$ as the angle between the dipole of a water molecule and the polarization of the external electric field, the ensemble average $\left\langle\cos ^{2}(\theta)\right\rangle$ was used as a measure of 
the water molecular alignment [22]. If water molecules are randomly oriented, $\left\langle\cos ^{2}(\theta)\right\rangle$ is equal to $1 / 3$. On the other hand, $\left\langle\cos ^{2}(\theta)\right\rangle$ becomes smaller than $1 / 3$ when water molecules are aligned perpendicular to the external electric field and larger than $1 / 3$ for parallel alignment. The timedependent $\left\langle\cos ^{2}(\theta)\right\rangle-1 / 3$ of the simulated water molecules is shown in Fig. 3(b) and compared with the experimental time-dependent anisotropy. The value of $\left\langle\cos ^{2}(\theta)\right\rangle$ is initially $\sim 0.009$ right after time zero, indicating that water molecules are aligned along with the electric field, and decays to zero corresponding to randomization. When $\left\langle\cos ^{2}(\theta)\right\rangle-1 / 3$ is convoluted with the IRF ( $\left.210 \mathrm{fs}\right)$, it exhibits qualitatively good agreement with the experiment. The time-dependent change of $\left\langle\cos ^{2}(\theta)\right\rangle-1 / 3$ is fitted with the biexponential decay with the time constants of 0.16 and 1.4 ps. The time constant of the fast component in MD matches that of experiment, while the slow component is slower in MD, whereas the relative contribution of the slow component to the total signal is larger in MD than in the experiment (see Supplemental Material [22], Tables S1 and S2). The current observations are consistent with previous $\mathrm{x}$-ray speckle visibility spectroscopy studies on liquid water showing that the decay of the structural correlation can be slower in MD than in the experimental data [32].

In order to investigate the effect of the electric field on local coordination, $\mathrm{O}-\mathrm{O}$ radial distribution functions $g_{\mathrm{OO}}(r)$ were calculated from the MD simulations and the results at $-1 \mathrm{ps}$ (before the electric field), $50 \mathrm{fs}$ (right after the electric field), and $2 \mathrm{ps}$ (after reorientation) are compared in Figs. 3(c) and 3(d). At 50 fs, the first peak at $2.8 \AA$ A becomes broader and the values at shorter distances are significantly increased, indicating that the first-neighbor distance is decreased due to alignment. The changes at 2 ps correspond exactly to heating, which is consistent with our experimental observation showing that the energy deposited by the pump pulse is converted to heat on a picosecond timescale by gradual energy relaxation. As can be seen in Fig. 3(d), the changes at 2 ps mainly affect the second coordination shell at distances $r=4.5 \AA$, which corresponds to tetrahedral coordination and shifts as a function of temperature [33].

It is hypothesized that liquid water can exist in two fluctuating forms of different density, namely, high- and low-density liquid [34-37]. This hypothesis has received both experimental [38-40] and theoretical [41] support and implies that liquid water can be understood as a fluctuating mixture of HDL and LDL. The hydrogen bond network of LDL species exhibits local tetrahedral coordination, whereas the environment around HDL species is more disordered. By using OKE spectroscopy Taschin et al. provided evidence of the coexistence of HDL and LDL local configurations in the low-frequency range $\sim 200 \mathrm{~cm}^{-1}$ [18]. Motivated by this approach, we analyzed our simulation in order to identify any differences on the OKE signal of two different local instantaneous structures of water
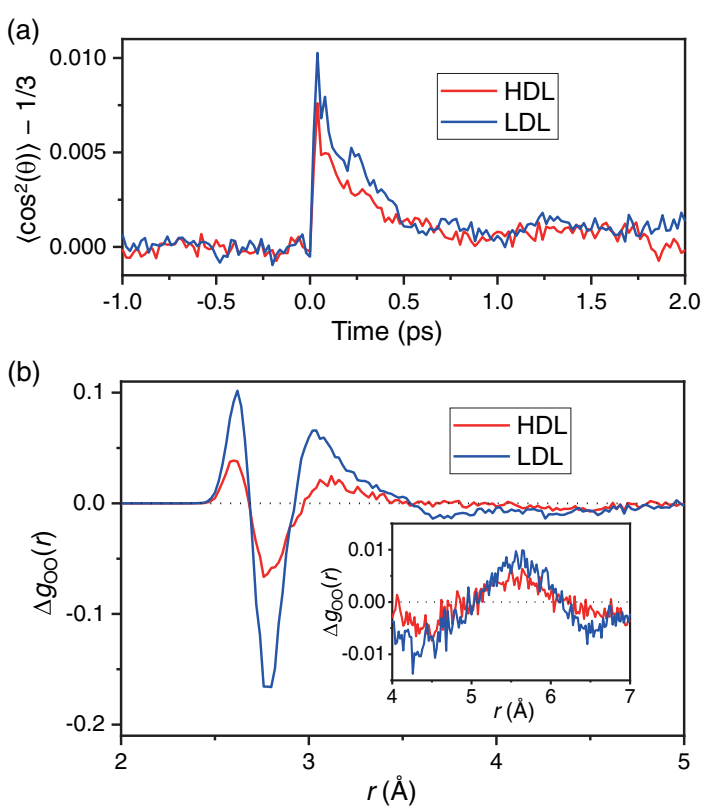

FIG. 4. LSI-dependent analysis. (a) Time-dependent $\left\langle\cos ^{2}(\theta)\right\rangle-1 / 3$ of HDL species (red) and LDL species (blue) are shown. The LDL species are more susceptible to alignment than HDL species, indicated by the higher amplitude. (b) Difference between the averaged oxygen-oxygen partial radial distribution functions for the time range from 0.0 to $0.5 \mathrm{ps}$ and the reference ( -1 to $-0.5 \mathrm{ps}$ ) for the HDL and LDL species. Again here, the LDL species are affected more by the electric field than HDL species, indicated by larger difference signal. (Inset) Details of the curves at larger distances.

based on direct structural information obtained by $\mathrm{x}$ rays. In order to differentiate between HDL and LDL contributions, we utilized the local structure index (LSI). The LSI is an order parameter representing how well the first and second coordination shells of water are separated and has been used as an indicator of transient structural fluctuations of water [42-45]. We performed LSI-dependent analysis on our simulations by following the definition given in previous studies [43-45] and the detailed procedure is given in the Supplemental Material [22].

The time-dependent $\left\langle\cos ^{2}(\theta)\right\rangle-1 / 3$ of $\mathrm{HDL}$ and LDL species are calculated separately and compared in Fig. 4(a). Two curves exhibit very similar decay constants at ambient conditions, consistent with previous theoretical studies probing dynamics with the LSI [46], but have different initial amplitudes indicating that LDL species are transiently oriented more than HDL species. The LSI-dependent partial O-O radial distribution functions $g_{\mathrm{OO}}(r)$ are also calculated from the MD simulation. The differences between the averaged $g_{\mathrm{OO}}(r)$ at the time delays ranging from 0.0 to $0.5 \mathrm{ps}$ and the reference ( -1 to $-0.5 \mathrm{ps}$ ) for HDL and LDL species are compared in Fig. 4(b). The $\Delta g_{\mathrm{OO}}(r)$ of LDL species has much larger amplitude, indicating that LDL species are influenced more by the electric field than the HDL species, which is 
consistent with the result from the time trace of the LSI-dependent $\left\langle\cos ^{2}(\theta)\right\rangle$. This observation can be attributed to the presence of stronger hydrogen bonds in LDL species [47], leading to a more delocalized wave function of the electronic structure than the corresponding HDL species [48] and as such resulting in increased polarizability. This observation is consistent with the fact that the dielectric constant of liquid water, which can be considered as a macroscopic measure of the polarizability, increases significantly upon cooling [49] and thus correlates with increasing LDL population.

In conclusion, by utilizing anisotropic $\mathrm{x}$-ray scattering we are able to probe the ultrafast structural dynamics of liquid water and can separate the anisotropic scattering contribution from the delayed temperature increase of $\sim 0.1 \mathrm{~K}$. The anisotropic decay occurs within $\sim 160$ fs and is attributed to loss of the intermolecular alignment imposed by the pump pulse polarization. The experiments are complemented by MD simulations with explicit electric field to simulate the effect of induced dipoles under fast oscillating fields. Both the experimental anisotropic scattering patterns and the corresponding time trace are well reproduced by the MD simulations and show that water molecules are aligned along the pump pulse polarization. The LSI-dependent analysis indicates that the two hypothesized transient structures, HDL and LDL, exhibit different susceptibilities toward the alignment imposed by the external field due to variation in local hydrogen bond strength.

Finally, we note that this approach is an important step toward being able to measure the dynamics of deeply supercooled water. Theoretical investigations indicate that, at temperatures near the Widom line, dynamical heterogeneity exhibits a maximum that can be associated with the fragile-to-strong transition [50], leading to changes in the corresponding diffusion constant [51]. Therefore, by using a combination of ultrafast anisotropic $\mathrm{x}$-ray scattering presented here with the evaporative cooling approach $[33,38,32,52]$, it is, in principle, possible to measure atomic-level intermolecular dynamics of water down to $227 \mathrm{~K}$.

The authors would like to acknowledge K. T. Wikfeldt for advice and useful discussions on the molecular dynamic simulations. This work has been supported by the European Research Council (ERC) Advanced Grant under Project No. 667205, the Swedish National Research Council (VR) under Grants No. 2013-8823, No. 201705128, and No. 2019-05542. This work is also supported by the National Research Foundation of Korea (NRF) grant funded by the Korea government (MSIT) (Grant No. 2019K1A3A1A47000562). The experiments were performed at beamline BL3:EH2 of SACLA with the approval of the Japan Synchrotron Radiation Research Institute (Proposals No. 2015A8038 and No. 2016B8055). *kimkyunghwan@ postech.ac.kr

f.perakis@fysiks.su.se

[1] S. T. Roberts, K. Ramasesha, and A. Tokmakoff, Acc. Chem. Res. 42, 1239 (2009).

[2] H. J. Bakker and J. L. Skinner, Chem. Rev. 110, 1498 (2010).

[3] F. Perakis, L. D. Marco, A. Shalit, F. Tang, Z. R. Kann, T. D. Kühne, R. Torre, M. Bonn, and Y. Nagata, Chem. Rev. 116, 7590 (2016).

[4] R. Righini, Science 262, 1386 (1993).

[5] N. T. Hunt, L. Kattner, R. P. Shanks, and K. Wynne, J. Am. Chem. Soc. 129, 3168 (2007).

[6] Q. Zhong and J. T. Fourkas, J. Phys. Chem. B 112, 15529 (2008).

[7] S. C. Santucci, D. Fioretto, L. Comez, A. Gessini, and C. Masciovecchio, Phys. Rev. Lett. 97, 225701 (2006).

[8] P. Gallo, F. Sciortino, P. Tartaglia, and S.-H. Chen, Phys. Rev. Lett. 76, 2730 (1996).

[9] G. Ruocco, F. Sette, U. Bergmann, M. Krisch, C. Masciovecchlo, V. Mazzacurati, G. Signorelli, and R. Verbeni, Nature (London) 379, 521 (1996).

[10] M. W. Evans, Phys. Lett. A 158, 216 (1991).

[11] M. S. Skaf and M. T. Sonoda, Phys. Rev. Lett. 94, 137802 (2005).

[12] M. T. Sonoda, S. M. Vechi, and M. S. Skaf, Phys. Chem. Chem. Phys. 7, 1176 (2005).

[13] P.-H. Tang and T.-M. Wu, J. Mol. Liq. 301, 112363 (2020).

[14] S. Palese, L. Schilling, R. J. D. Miller, P. R. Staver, and W. T. Lotshaw, J. Phys. Chem. 98, 6308 (1994).

[15] E. O. Potma, W. P. de Boeij, and D. A. Wiersma, Biophys. J. 80, 3019 (2001).

[16] R. Torre, P. Bartolini, and R. Righini, Nature (London) 428, 296 (2004).

[17] D. A. Turton and K. Wynne, J. Chem. Phys. 131, 201101 (2009).

[18] A. Taschin, P. Bartolini, R. Eramo, R. Righini, and R. Torre, Nat. Commun. 4, 2401 (2013).

[19] E. Biasin et al., Phys. Rev. Lett. 117, 013002 (2016).

[20] T. Ishikawa et al. Nat. Photonics 6, 540 (2012).

[21] T. Sato, T. Togashi, K. Ogawa, T. Katayama, Y. Inubushi, K. Tono, and M. Yabashi, Appl. Phys. Express 8, 012702 (2015).

[22] See Supplemental Material at http://link.aps.org/ supplemental/10.1103/PhysRevLett.125.076002 for details on the data collection, data processing, MD simulations, and LSI analysis.

[23] J. Li, Z. Nie, Y. Y. Zheng, S. Dong, and Z.-H. Loh, J. Phys. Chem. Lett. 4, 3698 (2013).

[24] K. H. Kim et al., Nature (London) 518, 385 (2015).

[25] J. Savolainen, S. Ahmed, and P. Hamm, Proc. Natl. Acad. Sci. U.S.A. 110, 20402 (2013).

[26] K. Ramasesha, L. De Marco, A. Mandal, and A. Tokmakoff, Nat. Chem. 5, 935 (2013).

[27] L. B. Skinner, C. J. Benmore, B. Shyam, J. K. R. Weber, and J. B. Parise, Proc. Natl. Acad. Sci. U.S.A. 109, 16463 (2012).

[28] P. Zalden, L. Song, X. Wu, H. Huang, F. Ahr, O. D. Mücke, J. Reichert, M. Thorwart, P. K. Mishra, R. Welsch, R. Santra, F. X. Kärtner, and C. Bressler, Nat. Commun. 9, 2142 (2018). 
[29] G. Lamoureux, E. Harder, I. Vorobyov, B. Roux, and A. D. MacKerell, Chem. Phys. Lett. 418, 245 (2006).

[30] C. Caleman and D. van der Spoel, Angew. Chem., Int. Ed. Engl. 47, 1417 (2008).

[31] M. J. Abraham, T. Murtola, R. Schulz, S. Páll, J. C. Smith, B. Hess, and E. Lindahl, SoftwareX 1-2, 19 (2015).

[32] F. Perakis et al., Nat. Commun. 9, 1917 (2018).

[33] J. A. Sellberg et al., Nature (London) 510, 381 (2014).

[34] P. H. Poole, F. Sciortino, U. Essmann, and H. E. Stanley, Nature (London) 360, 324 (1992).

[35] C. A. Angell, Nat. Mater. 13, 673 (2014).

[36] A. Nilsson and L. G. M. Pettersson, Nat. Commun. 6, 8998 (2015).

[37] P. Gallo, K. Amann-Winkel, C. A. Angell, M. A. Anisimov, F. Caupin, C. Chakravarty, E. Lascaris, T. Loerting, A. Z. Panagiotopoulos, J. Russo, J. A. Sellberg, H. E. Stanley, H. Tanaka, C. Vega, L. Xu, and L. G. M. Pettersson, Chem. Rev. 116, 7463 (2016).

[38] K. H. Kim, A. Späh, H. Pathak, F. Perakis, D. Mariedahl, K. Amann-Winkel, J. A. Sellberg, J. H. Lee, S. Kim, J. Park, K. H. Nam, T. Katayama, and A. Nilsson, Science 358, 1589 (2017).

[39] F. Perakis, K. Amann-Winkel, F. Lehmkühler, M. Sprung, D. Mariedahl, J. A. Sellberg, H. Pathak, A. Späh, F. Cavalca, D. Schlesinger, A. Ricci, A. Jain, B. Massani, F. Aubree, C. J. Benmore, T. Loerting, G. Grübel, L. G. M. Pettersson, and A. Nilsson, Proc. Natl. Acad. Sci. U.S.A. 114, 8193 (2017).
[40] S. Woutersen, B. Ensing, M. Hilbers, Z. Zhao, and C. A. Angell, Science 359, 1127 (2018).

[41] J. C. Palmer, R. Car, and P. G. Debenedetti, Faraday Discuss. 167, 77 (2014).

[42] E. Shiratani and M. Sasai, J. Chem. Phys. 104, 7671 (1996).

[43] E. Shiratani and M. Sasai, J. Chem. Phys. 108, 3264 (1998).

[44] K. T. Wikfeldt, A. Nilsson, and L. G. M. Pettersson, Phys. Chem. Chem. Phys. 13, 19918 (2011).

[45] D. Schlesinger, K. T. Wikfeldt, L. B. Skinner, C. J. Benmore, A. Nilsson, and L. G. M. Pettersson, J. Chem. Phys. 145, 084503 (2016).

[46] G. Camisasca, N. Galamba, K. T. Wikfeldt, and L. G. M. Pettersson, J. Chem. Phys. 150, 224507 (2019).

[47] A. Nilsson and L. G. M. Pettersson, Chem. Phys. 389, 1 (2011).

[48] D. Nordlund, H. Ogasawara, H. Bluhm, O. Takahashi, M. Odelius, M. Nagasono, L. G. M. Pettersson, and A. Nilsson, Phys. Rev. Lett. 99, 217406 (2007).

[49] J. D. Bernal and R. H. Fowler, J. Chem. Phys. 1, 515 (1933).

[50] R. Shi, J. Russo, and H. Tanaka, Proc. Natl. Acad. Sci. U.S.A. 115, 9444 (2018).

[51] Y. Ni, N. J. Hestand, and J. L. Skinner, J. Chem. Phys. 148, 191102 (2018).

[52] K. H. Kim, H. Pathak, A. Späh, F. Perakis, D. Mariedahl, J. A. Sellberg, T. Katayama, Y. Harada, H. Ogasawara, L. G. M. Pettersson, and A. Nilsson, Phys. Rev. Lett. 119, 075502 (2017). 